\title{
Impaired Expression of Cardiac Adiponectin in Leptin- Deficient Mice With Viral Myocarditis
}

\author{
Takashi TAKAHASHI, ${ }^{1}$ MD, Fei YU, ${ }^{1} \mathrm{MD}$, Seiichiro SAEguSA, ${ }^{1} \mathrm{MD}$, \\ Hiroyuki Sumino, ${ }^{3}$ MD, Takeshi NAKAHASHI, ${ }^{2}$ MD, Kunimitsu IwAI, ${ }^{2}$ MD, \\ Shigeto MORIMOTO, ${ }^{2} \mathrm{MD}$, Masahiko KURABAYASHI, ${ }^{3} \mathrm{MD}$, \\ and Tsugiyasu KANDA, ${ }^{1} \mathrm{MD}$
}

\begin{abstract}
SUMMARY
A mouse model of encephalomyocarditis (EMC) virus-induced myocarditis was used to investigate the expression of adiponectin in damaged cardiomyocytes. We intraperitoneally injected EMC virus into leptin-deficient $o b / o b(\mathrm{OB})$ mice and wild-type (WT) mice. OB mice were divided into two subgroups consisting of mice with no intervention and mice receiving leptin replacement starting simultaneously with viral inoculation. We determined differences in heart weight, cardiac histological score, numbers of infiltrating and apoptotic cells in the myocardium, expression levels of adiponectin and TNF- $\alpha$ mRNA in the heart, adiponectin immunoreactivity in myocytes, adiponectin and TNF- $\alpha$ concentrations in the heart, and immunoreactivity of adiponectin receptors in myocytes between OB mice and WT mice. There was significantly decreased adiponectin mRNA expression, immunoreactivity, and protein level in the heart, and reduced immunoreactivity of adiponectin receptor 1 in myocytes from OB mice on days 4 and 8 after viral inoculation as compared with those in WT mice, together with increased cardiac weight, severe inflammatory myocardial damage, and increased levels of cardiac TNF- $\alpha$ mRNA and protein. Replacement of leptin in OB mice inhibited the development of severe myocarditis through augmentation of adiponectin mRNA, immunoreactivity, and protein level, increased adiponectin receptor 1 immunoreactivity in myocytes, and suppressed levels of TNF- $\alpha$ mRNA and protein. These results suggest that impaired expression of cardiac adiponectin may contribute to the progression of viral myocarditis through enhanced expression of TNF- $\alpha$ under a leptin-deficient condition. (Int Heart J 2006; 47: 107-123)
\end{abstract}

Key words: Adiponectin, Leptin deficiency, Viral myocarditis, Cardiomyocyte

HEART failure is generally considered to begin with myocyte damage caused by a variety of pathological conditions that include ischemia, toxins, and myocardial infection. The heart compensates by dilatation and cellular hypertrophy, and eventually decompensates, resulting in heart failure. A proinflammatory cytok-

From the Departments of ${ }^{1}$ General Medicine and ${ }^{2}$ Geriatric Medicine, Kanazawa Medical University, Ishikawa and

${ }^{3}$ Department of Medicine and Biological Science, Gunma University Graduate School of Medicine, Gunma, Japan.

Address for correspondence: Tsugiyasu Kanda, MD, Department of General Medicine, Kanazawa Medical University, 1-1

Daigaku, Uchinada-machi, Kahoku-gun, Ishikawa 920-0293, Japan.

Received for publication January 24, 2005

Revised and accepted May 9, 2005. 
ine, tumor necrosis factor- $\alpha$ (TNF- $\alpha$ ), has recently been postulated to be one of the pathogenetic factors responsible for the progression from compensated to decompensated heart failure. Mann and colleagues in 1996 demonstrated that the nonfailing human heart does not express, whereas the failing human heart expresses a significant amount of TNF- $\alpha \cdot{ }^{1)}$ Moreover, TNF- $\alpha$ immediately inhibits the contractility of isolated cardiac myocytes in a dose-dependent manner, and this negative inotropic action is completely reversible upon the removal of TNF$\alpha^{2)}$

Identification of leptin is a good example of a contribution made by molecular biology to understanding the mechanisms initially hypothesized from classic physiological studies. ${ }^{3)}$ Several papers have focused on the association between leptin and cardiovascular disease, such as hypertension and cachexia, in chronic heart failure. ${ }^{4-6)}$ A reduced leptin concentration may diminish the degree of cardiac adaptation to heart failure. ${ }^{7)}$ It has also been shown that plasma leptin levels are inappropriately low in patients with cachectic chronic heart failure. ${ }^{5)}$ We have recently found that leptin deficiency enhances myocardial necrosis and lethality in a mouse model of viral myocarditis, suggesting a protective action of leptin against myocyte damage ${ }^{8)}$ However, it is still unclear how severe inflammatory myocardial injury induced by viral infection develops under leptin deficiency.

Adiponectin, also known as $30-\mathrm{kDa}$ adipocyte complement-related protein, ${ }^{9)}$ is a hormone secreted by adipocytes that acts as an antidiabetic and antiatherogenic cytokine. ${ }^{10)}$ The concentration of adiponectin in blood is diminished under conditions of obesity, insulin resistance, and type II diabetes. ${ }^{9)}$ Adiponectin administration is reported to have glucose-lowering effects and to improve insulin resistance in mice. ${ }^{11)}$ On the other hand, adiponectin-deficient mice develop insulin resistance and diabetes. ${ }^{12)}$ This effect of adiponectin appears to be mediated by elevation of fatty acid oxidation through activation of AMP-activated protein kinase ${ }^{13)}$ and peroxisome proliferator-activated receptor (PPAR)- $\alpha{ }^{10)}$ In clinical research, plasma adiponectin concentrations in subjects with both diabetes mellitus and coronary artery disease have been shown to be lower than in patients with coronary artery disease alone. ${ }^{14)}$ Individuals with very low plasma adiponectin levels may be at increased risk of developing both diabetes mellitus and coronary artery disease.

Comparison of the organization of the adiponectin gene with that of the obese gene, which encodes leptin, shows several striking similarities in humans. ${ }^{15)}$ These two genes, which are composed of three exons and have a long first intron, are expressed specifically in adipose tissue. ${ }^{15)}$ Adiponectin and leptin control fuel homeostasis, body weight, and insulin sensitivity. In another recent study, amelioration of insulin resistance, pancreatic $\beta$-cell degranulation, and diabetes after crossing leptin-deficient mice with globular domain adiponectin trans- 
genic mice has been described, indicating that globular adiponectin and leptin may have overlapping functions. ${ }^{10)}$ Thus, adiponectin may also possess a function similar to the crucial role of leptin in the development of heart failure.

We hypothesized that cardiac expression of adiponectin could play a protective role against the progression of severe viral myocarditis under a leptin-deficient status. Therefore, we examined cardiac adaptation to heart failure through adiponectin expression in leptin-deficient mice with acute viral myocarditis, and the influence of leptin replacement therapy on adiponectin expression in the myocardium of leptin-deficient mice.

\section{MATERIALS AND METHODS}

Animals: Six-week-old female leptin-deficient ob/ob (OB) mice and C57BL wild-type (WT) mice were obtained from Jackson Laboratory (Bar Harbor, Maine, USA).

Virus: A myocarditic variant of encephalomyocarditis (EMC) virus was obtained from Y. Seto, PhD (Keio University, Tokyo, Japan). Virus preparations were stored at $-80^{\circ} \mathrm{C}$ in Eagle's minimum essential medium supplemented with $0.1 \%$ fetal bovine serum until the time of use.

Infection protocol: Animals were intraperitoneally inoculated with 500 plaqueforming units of EMC virus suspended in $0.1 \mathrm{~mL}$ of saline.

Treatment protocol: WT $(n=10)$ and OB $(n=40)$ mice were injected with EMC virus. In addition, $\mathrm{OB}$ mice were randomly assigned to one of two groups. The first group $(n=20)$ received no interventional therapy. The second group $(n=20)$ of OB mice received a daily intraperitoneal injection of recombinant mouse leptin (300 $\mu \mathrm{g}$ per day, starting simultaneously with EMC virus inoculation, OB + Lep group) ${ }^{8)}$ Cardiac tissues were immediately extracted after sacrifice by cervical dislocation on days 4 and 8 after viral inoculation.

Histological examinations of hearts: Body weight was recorded before sacrifice. The heart was immediately weighed after sacrifice. Half of the cardiac tissue was fixed in $10 \%$ buffered formalin and stained with hematoxylin-eosin $(\mathrm{H} \& \mathrm{E})$, while the other half was immediately frozen in liquid nitrogen and stored at $-80^{\circ} \mathrm{C}$ for cytokine analyses. Two transverse sections of the ventricular myocardium were graded for the severity of necrosis and mononuclear cell infiltration by an experienced pathologist, who had no knowledge of the study design, according to the following scale: grade 1 , lesions involving $<25 \%$ of the ventricular myocardium; grade 2, lesions involving 25 to $50 \%$ of the myocardium; grade 3, lesions involving 50 to $75 \%$ of the myocardium; and grade 4 , lesions involving $>75 \%$ of the myocardium. We also performed staining of myosin to identify myocyte necrosis accurately. In addition, the pathologist randomly selected 5 
high power fields $(\mathrm{HPF})(\times 400$ magnification) from each transverse section of the myocardium, and counted the infiltrating cells. The number of apoptotic cells in 5 randomly selected HPF $(\times 400$ magnification) per section in the transverse sections of myocardium was determined by in situ TUNEL as previously described. ${ }^{16)}$

Detection of adiponectin mRNA in cardiomyocytes: In situ hybridization (ISH) using Digoxigenin (DIG) REMBRANDT for DNA ISH and a detection kit (Code HKD38003, Pan Path Co. Ltd., Amsterdam, Netherlands) was performed on serial sections of the heart from various mice on day 8 after viral inoculation as previously described. ${ }^{17)}$ The presence of adiponectin mRNA was also examined in cardiac tissue from a normal wild-type mouse with neither viral inoculation nor leptin administration. We used commercially synthesized DIG-labeled mouse adiponectin sense and antisense RNA probes (Hokkaido System Science Co. Ltd., Hokkaido, Japan). Each section was hybridized with the labeled probes at $37^{\circ} \mathrm{C}$ for 2 hours. This was followed by several washes in Tris-buffer and RNase A solutions. The sections were then incubated with alkaline phosphatase-conjugated rabbit anti-DIG Fab fragments (\#D5105, DAKO Cytomation Co. Ltd., Kyoto, Japan). Signals were visualized with 4-nitro-blue-tetrazolium and 5bromo-4-chloro-3-indolyl phosphate. The slides were blindly reviewed by the same pathologist, and the hybridization signal of adiponectin mRNA was determined for myocytes.

Comparative expression levels of adiponectin and TNF- $\alpha$ mRNA in cardiac tissues: RNA extraction was performed on half of each frozen cardiac tissue specimen as specified by the manufacturer (RNeasy Mini Kit, QIAGEN Inc., Tokyo). Total RNA concentration was determined by measuring the optical density at $260 \mathrm{~nm}$. Aliquots of $20 \mu \mathrm{L}$ of RNA from each tissue sample were used for the production of cDNA. Comparative expression levels of adiponectin mRNA in cardiac tissue from different groups were determined using quantitative real-time reverse transcriptase-polymerase chain reaction (RT-PCR) as described previously. ${ }^{18)}$ The expression levels of TNF- $\alpha$ mRNA were also examined by quantitative real-time RT-PCR. We used a commercially available kit for TNF- $\alpha$ and adiponectin RT-PCR (Mm00443258 mL and Mm00456425 mL, respectively, Applied Biosystems Inc., Foster City, CA, USA). The optimal number of cycles of RT-PCR was examined for the adiponectin mRNA level. The glyceraldehyde3-phosphate dehydrogenase (GAPDH) gene was used as an endogenous internal standard, and was amplified with specific primers for the number of cycles. The gradient of the cycle numbers for adiponectin to GAPDH was determined. Adiponectin mRNA levels in the heart were calculated as comparative values, which were normalized to adiponectin mRNA in the heart from a normal wild-type mouse with neither viral inoculation nor leptin administration (assigned value 
equal to 1).

Adiponectin immunoreactivity in cardiomyocytes: Immunohistochemical staining using the streptavidin biotin complex method (\#K0675 and \#E0353, DAKO Cytomation) was performed on serial transverse sections of ventricular myocardium from different mice on days 4 and 8 after viral inoculation. As a normal control, adiponectin immunoreactivity was determined in a heart from a normal wildtype mouse with neither viral inoculation nor leptin administration. We used the following commercially available primary antibody at a dilution of 1:50; rabbit polyclonal anti-mouse adiponectin antibody (\#ACRP303-A, Alpha Diagnostic International Inc., San Antonio, TX, USA). Control slides were treated with normal diluted rabbit serum. The slides were blindly reviewed by the same pathologist, and were semiquantitatively graded according to the degree of immunoreactivity: 0 for absence of staining, $1+$ for weak, $2+$ for moderate, and $3+$ for strong staining. ${ }^{19)}$ They were compared with the respective control slides to exclude nonspecific staining. Adiponectin immunoreactivity was assessed in 30 randomly selected myocytes corresponding to the surviving cells found in respective $\mathrm{H} \& \mathrm{E}$ - and myosin-stained slides.

Concentrations of adiponectin and TNF- $\alpha$ in heart: Adiponectin and TNF- $\alpha$ levels in the homogenate of each tissue were measured using the other half of frozen cardiac tissue samples. An enzyme-linked immunosorbent assay (ELISA), which used a polyclonal antibody specific for mouse TNF- $\alpha$ or adiponectin precoated onto a microtiter plate (ELISA kit for TNF- $\alpha$. BioSource International Inc., Camarillo, CA, USA; ELISA kit for adiponectin: Otsuka Pharmaceutical Co., Ltd., Tokyo), was performed on tissue samples according to the manufacturers' instructions. As a normal control, cardiac levels of adiponectin and TNF- $\alpha$ were determined in the heart from a normal WT mouse without viral infection. For data processing, we allocated the minimum values detected by the ELISA assay to all samples with concentrations below the detection threshold. The ELISA kit used for TNF- $\alpha$ concentration showed that the limit of sensitivity and the intra- and interassay variations were $3.0 \mathrm{pg} / \mathrm{mL}, 6.5 \%$, and $8.7 \%$, respectively. The ELISA kit used for adiponectin levels demonstrated sensitivity, intra-assay variance, and cross-reactivity of $0.25 \mathrm{ng} / \mathrm{mL}$, less than $10 \%$, and no response for specimens from other animals including sheep, respectively.

Immunoreactivity of adiponectin receptors in cardiomyocytes: To examine the immunoreactivity of adiponectin receptors 1 and 2 (AdipoR 1 and AdipoR2) in the heart, immunohistochemical staining by the streptavidin biotin complex method was performed on serial sections of myocardium on days 4 and 8, which had been used to determine the adiponectin immunoreactivity of myocytes. The immunoreactivity of AdipoR1 and AdipoR2 in vessels and macrophages was considered as a positive control. As a normal control, the immunoreactivity of 
AdipoR1 or AdipoR2 was determined in the heart from a normal wild-type mouse with neither viral inoculation nor leptin administration. We used the following primary antibodies at a dilution of 1:50: rabbit polyclonal anti-mouse AdipoR1 or AdipoR2 antibodies (\#ADIPOR11-A or ADIPOR21-A, Alpha Diagnostic International Inc.). Control slides were treated with normal diluted rabbit serum. The slides were blindly reviewed by the same pathologist, and were semiquantitatively graded according to the criteria for immunoreactivity as used for adiponectin immunoreactivity. They were compared with the respective control slides, and AdipoR1 or AdipoR2 immunoreactivity was evaluated in 30 myocytes corresponding to cells where adiponectin immunoreactivity was observed.

Statistical analyses: Data are expressed as the mean \pm standard deviation. Analysis of variance (ANOVA) was used to evaluate the differences in body and cardiac weights, numbers of infiltrating and apoptotic cells in the myocardium, comparative expression level of adiponectin mRNA in cardiac tissue, and adiponectin concentration in the heart, as compared with those in the WT group. The Kruskal-Wallis test was used for nonparametric analysis to assess the differences in cardiac histological score and immunoreactivity of adiponectin or its receptors in myocytes. A value of $P<0.05$ was considered to be statistically significant.

\section{RESULTS}

Mortality in different mice with viral myocarditis: The numbers of mice with viral myocarditis that died were 1, 9, and 2 in the WT, OB, and OB + Lep groups, respectively, during the period of the treatment protocol. The numbers of mice in different groups from which heart specimens were obtained on days 4 and 8 after viral inoculation were 5 and 4 in the WT group, 6 and 5 in the OB group, and 10 and 8 in the $\mathrm{OB}+$ Lep group, respectively.

Body weight and cardiac weight: Body weight on days 0,4 , and 8 after viral infection was significantly greater in the $\mathrm{OB}$ and $\mathrm{OB}+$ Lep groups than in the WT group $(P<0.05$, Table). Heart weight in the OB group on day 8 after viral inocu-

Table. Body Weight and Cardiac Weight in Different Mice Groups After Viral Inoculation

\begin{tabular}{llllrl}
\hline & \multicolumn{2}{l}{ Body weight $(\mathrm{g})$} & \multicolumn{2}{c}{ Cardiac weight $(\mathrm{mg})$} \\
\cline { 2 - 6 } & Day 0 & Day 4 & Day 8 & Day 4 & Day 8 \\
\hline WT & $18.1 \pm 1.2$ & $18.3 \pm 1.5$ & $18.9 \pm 1.7$ & $97 \pm 5$ & $101 \pm 8$ \\
OB & $37.3 \pm 2.5^{*}$ & $37.1 \pm 2.9^{*}$ & $38.2 \pm 3.1^{*}$ & $105 \pm 7$ & $118 \pm 15^{*}$ \\
OB + Lep & $36.9 \pm 2.8^{*}$ & $37.2 \pm 3.5^{*}$ & $35.1 \pm 4.1^{*}$ & $99 \pm 8$ & $103 \pm 11$ \\
\hline
\end{tabular}

WT indicates wild-type mice; $\mathrm{OB}, \mathrm{ob} / \mathrm{ob}$ mice; and $\mathrm{OB} \pm$ Lep, ob/ob mice receiving leptin. Data are expressed as means $\pm \mathrm{SD}$. $* P<0.05$ compared with WT mice group. 


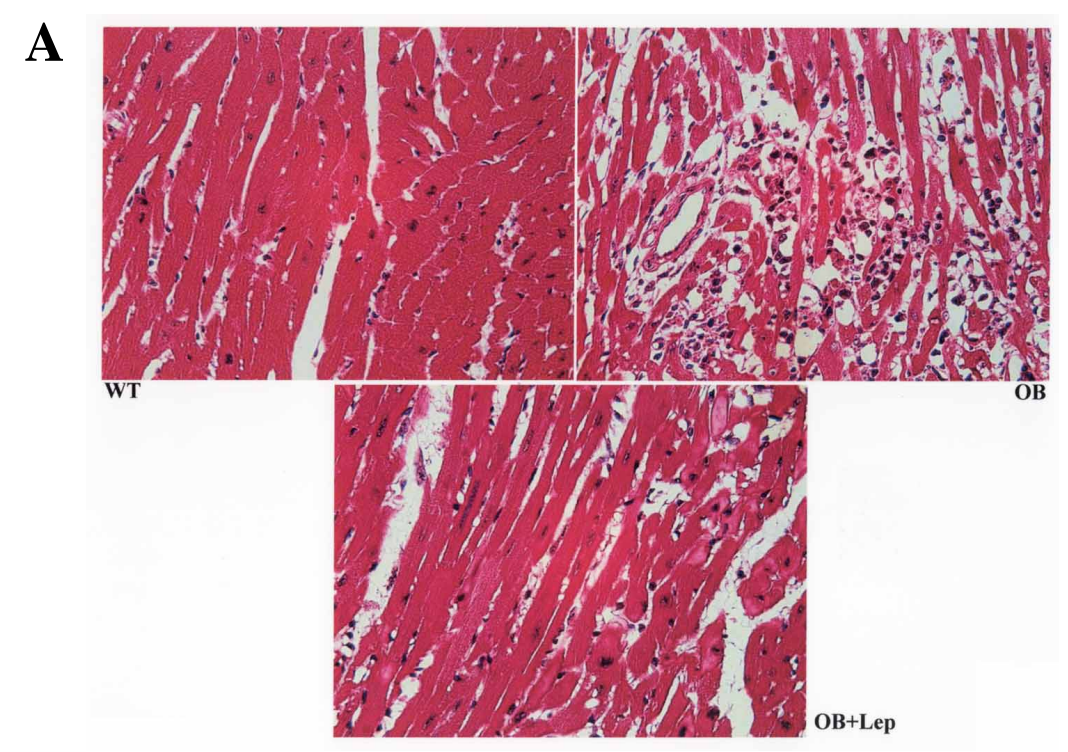

B

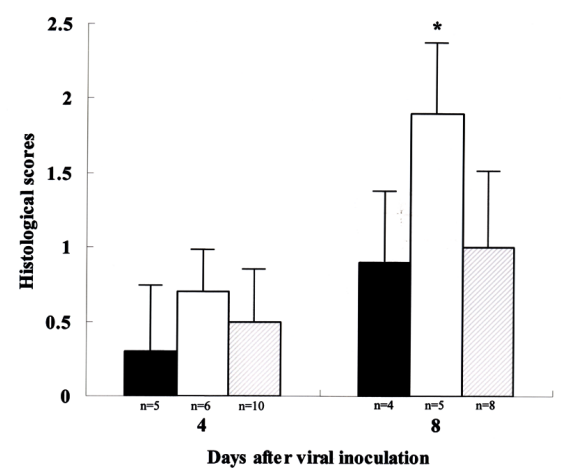

D

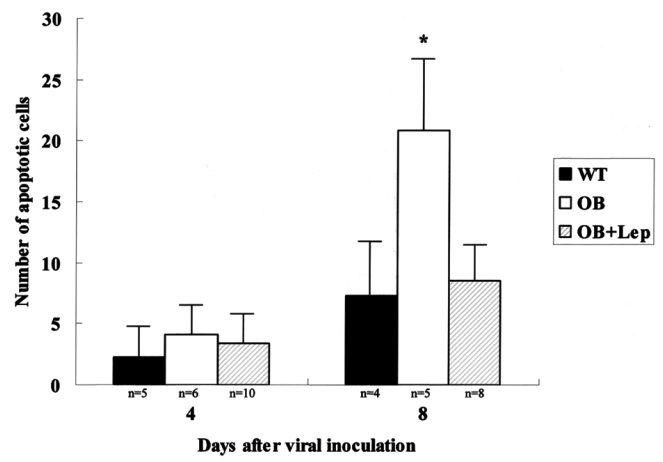

C

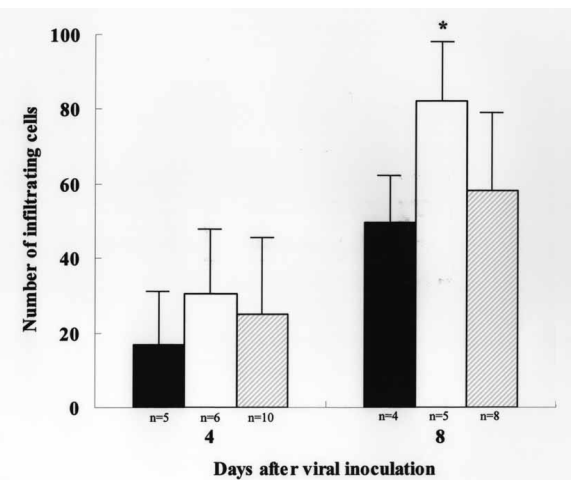

Figure 1. Photographic demonstration of histological findings in myocardium from various mice on day 8 after viral inoculation (A, original magnification). Results of histological examinations including histological score in hearts (B) and numbers of infiltrating (C) and apoptotic (D) cells in myocardium from different mice on days 4 and 8. Data are expressed as mean \pm SD. WT indicates wild-type mice; $\mathrm{OB}$, leptin-deficient ob/ob mice; and $\mathrm{OB}+\mathrm{Lep}, \mathrm{OB}$ mice receiving daily intraperitoneal administration of mouse leptin $(300 \mu \mathrm{g} /$ day, starting simultaneously with viral inoculation). $* P<0.05$ compared with WT group. 
lation was significantly increased as compared with that in the WT group $(P<$ 0.05 , Table). There was no significant difference in cardiac weight between the $\mathrm{OB}+$ Lep group and the WT group.

Histological findings in heart: Photographic demonstration of the histological findings in the myocardium from various mice on day 8 after virus infection is shown in Figure 1A. The histological score and numbers of infiltrating and apoptotic cells per field in hearts from different mice on days 4 and 8 are shown in Figures 1B, 1C, and 1D, respectively. The hearts from the OB group showed severe myocardial necrosis and mononuclear cell infiltration. The histological score for myocardial necrosis and cell infiltration on day 8 was significantly higher in the OB group than in the WT group $(P<0.05$, Figure $1 \mathrm{~B})$. The number of infiltrating cells per field in the ventricular myocardium on day 8 in the OB group was significantly elevated as compared with that in the WT group $(P<$ 0.05 , Figure 1C). The number of apoptotic cells per field in the heart on day 8 was significantly higher in the OB group than in the WT group $(P<0.05$, Figure 1D). There were no significant differences in histological score and numbers of infiltrating and apoptotic cells between the OB + Lep mice and WT mice.

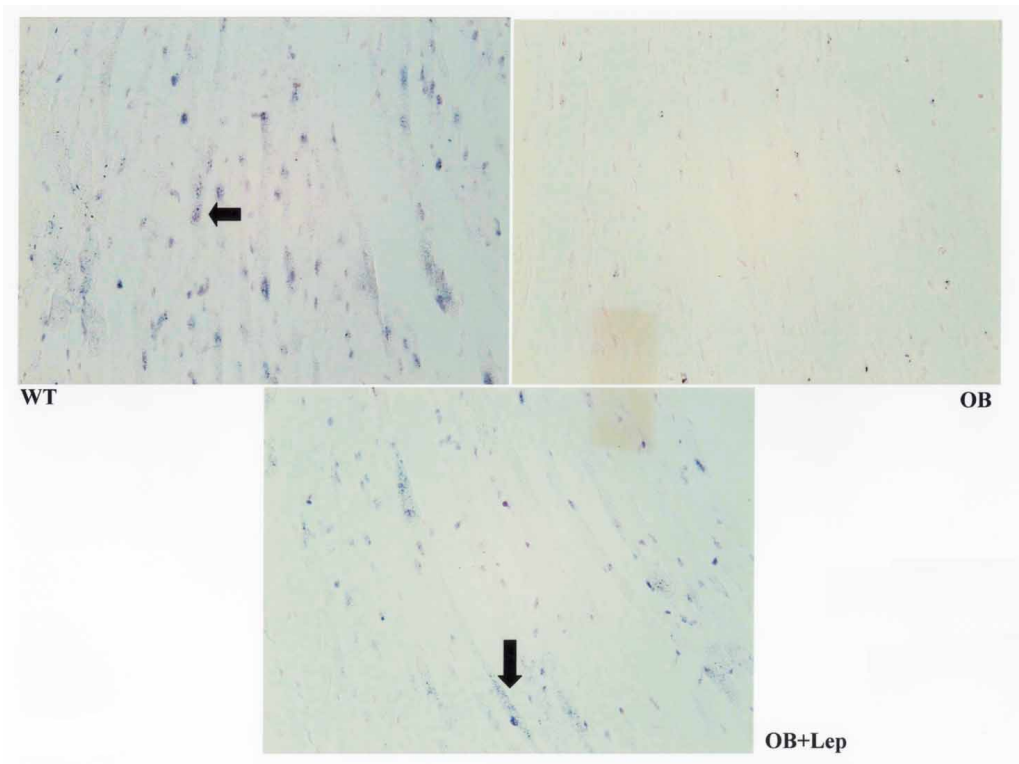

Figure 2. Detection of adiponectin mRNA (arrows) by in situ hybridization in myocardium from various mice on day 8 after viral infection. In situ hybridization using a digoxigenin-labeled adiponectin antisense riboprobe was performed on myocardial sections from different mice. WT indicates wild-type mice; OB, leptin-deficient ob/ob mice; and $\mathrm{OB}+$ Lep, $\mathrm{OB}$ mice receiving daily intraperitoneal administration of mouse leptin ( $300 \mu \mathrm{g} / \mathrm{day}$, starting simultaneously with viral inoculation). 
Detection of adiponectin mRNA in cardiomyocytes: To investigate the localization of adiponectin mRNA in the heart, ISH was performed on myocardial sections using an adiponectin antisense RNA probe. Adiponectin mRNA was not found in myocytes from a normal wild-type mouse. A moderate to strong signal for adiponectin mRNA was detected in myocytes from WT mice and OB + Lep mice on day 8 after viral infection (Figure 2). The adiponectin signal in the myocardium from $\mathrm{OB}$ mice at the same time was only very slight (Figure 2). There was no detectable adiponectin signal when ISH was performed on these sections using the sense probe.

Comparative expression levels of adiponectin and TNF- $\alpha$ mRNA in cardiac tissue: Comparative expression levels of adiponectin and TNF- $\alpha$ mRNA in the hearts from different mice on days 4 and 8 after virus infection are shown in Figures $3 \mathrm{~A}$ and $3 \mathrm{~B}$, respectively. Adiponectin mRNA levels in the hearts on days 4 and 8 were significantly lower in $\mathrm{OB}$ mice than in WT mice $(P<0.05$, Figure 3A). There was no difference in the cardiac levels of adiponectin mRNA between OB + Lep mice and WT mice. On the other hand, significantly elevated levels of TNF- $\alpha$ mRNA were observed on days 4 and 8 in the OB group compared with those in the WT group $(P<0.05$, Figure $3 \mathrm{~B})$. There was no significant difference in TNF- $\alpha$ mRNA levels between the OB + Lep group and WT group.

Adiponectin immunoreactivity in cardiomyocytes: Adiponectin immunoreactivity was not observed in the myocardium from a normal wild-type mouse. Photo-
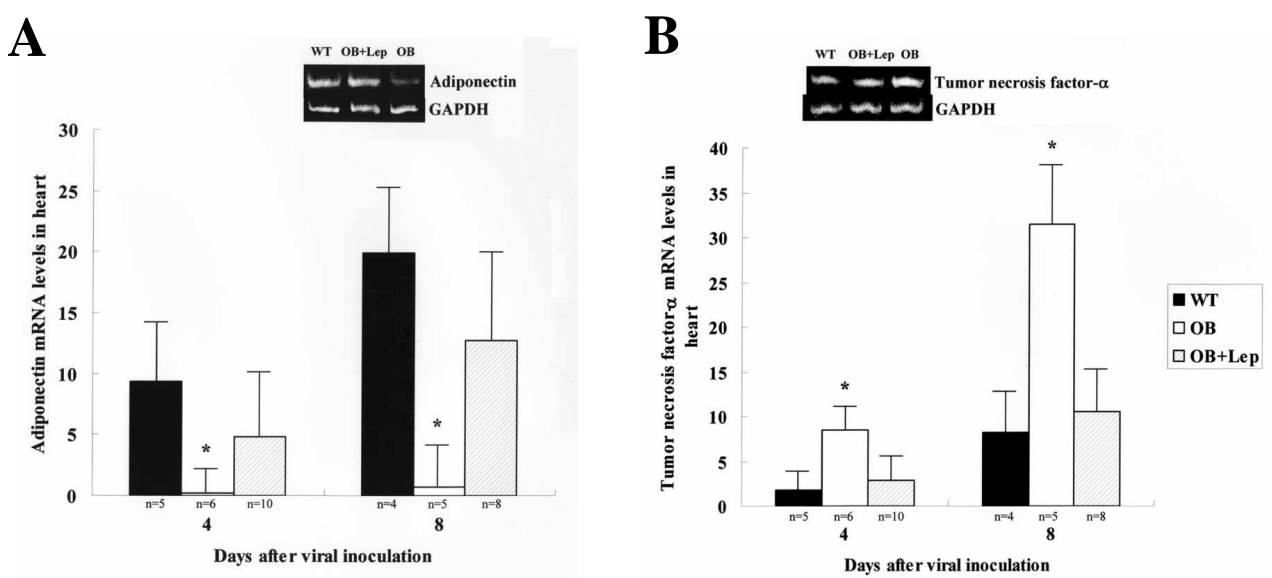

Figure 3. Comparative adiponectin (A) and tumor necrosis factor- $\alpha(\mathbf{B})$ mRNA levels using quantitative real-time reverse transcriptase-polymerase chain reaction with hearts from various mice on days 4 and 8 after viral infection, and demonstration of amplified cardiac adiponectin (A) and tumor necrosis factor- $\alpha(\mathbf{B})$ mRNA and corresponding glyceraldehyde-3-phosphate dehydrogenase gene on agarose gel, which were derived from various mice on day 8 after viral inoculation. Data are expressed as mean $\pm \mathrm{SD}$. WT indicates wild-type mice; $\mathrm{OB}$, leptin-deficient ob/ob mice; and $\mathrm{OB}+\mathrm{Lep}$, OB mice receiving daily intraperitoneal administration of mouse leptin (300 $\mu \mathrm{g} /$ day, starting simultaneously with viral inoculation). $* P<0.05$ compared with WT group. 


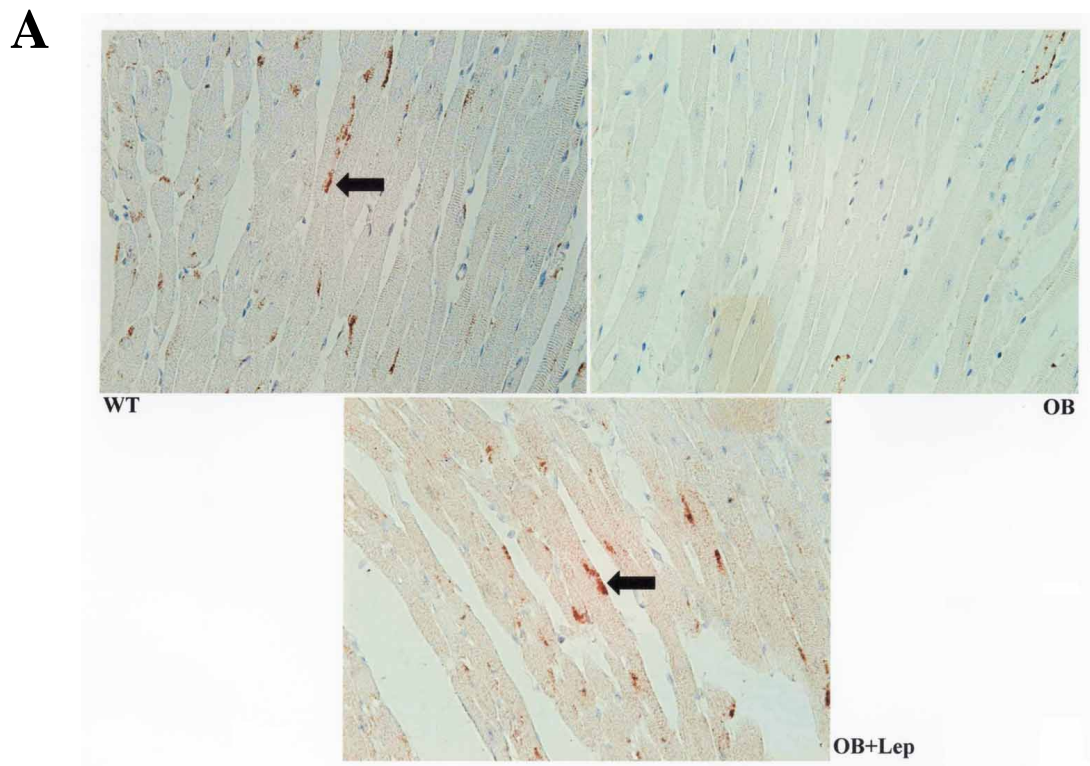

B

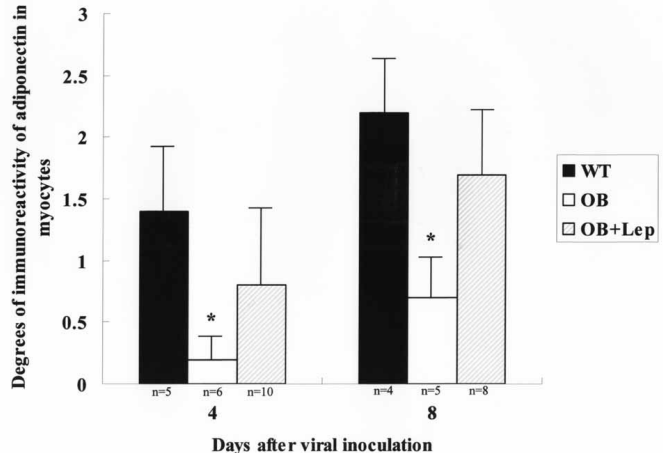

Figure 4. Adiponectin immunoreactivity in cardiomyocytes consisting of photographic demonstration of adiponectin immunoreactivity (arrows) in myocardium from different mice on day 8 after viral inoculation (A, original magnification) and adiponectin immunoreactivity in myocytes $(\mathbf{B})$ from various mice on days 4 and 8 . Data are expressed as mean \pm SD. WT indicates wild-type mice; $\mathrm{OB}$, leptin-deficient ob/ob mice; and $\mathrm{OB}+$ Lep, $\mathrm{OB}$ mice receiving daily intraperitoneal administration of mouse leptin (300 $\mu \mathrm{g} /$ day, starting simultaneously with viral inoculation). ${ }^{*} P<0.05$ compared with WT group.

graphic demonstration of immunoreactivity of adiponectin in myocytes from different mice on day 8 after viral inoculation is shown in Figure 4A. Adiponectin immunoreactivity in myocytes from various mice on days 4 and 8 is shown in Figure 4B. We found significantly suppressed adiponectin immunoreactivity in myocytes from the OB group as compared with those from the WT group at the 
same times $(P<0.05$, Figure 4B). There was expression of adiponectin in myocardial cells from $\mathrm{OB}+$ Lep mice, which was similar to the findings of adiponectin immunoreactivity in myocytes from WT mice. The immunoreactive distribution of adiponectin protein in transverse ventricular sections largely overlapped with that of adiponectin mRNA observed using the ISH method.

Concentrations of adiponectin and TNF- $\alpha$ in heart: Specimens of heart from a normal wild-type mouse showed undetectable levels of each molecule. Adiponectin and TNF- $\alpha$ concentrations in the hearts from different mice on days 4 and 8 after viral infection are shown in Figures 5A and 5B, respectively. Cardiac concentrations of adiponectin on days 4 and 8 were significantly lower in the OB group than in the WT group $(P<0.05$, Figure $5 \mathrm{~A})$. There was no difference in cardiac concentrations of adiponectin between the $\mathrm{OB}+$ Lep group and WT group. On the other hand, significantly increased concentrations of TNF- $\alpha$ were found on days 4 and 8 in OB mice compared with those in WT mice $(P<0.05$, Figure 5B). There was no significant difference in TNF- $\alpha$ concentrations between OB + Lep mice and WT mice.

Immunoreactivity of adiponectin receptors in cardiomyocytes: AdipoR1 and AdipoR2 immunoreactivity was found in the arterial wall and macrophages obtained from a normal wild-type mouse, respectively. Photographic demonstration of immunoreactivity of adiponectin receptor 1 in myocardium from various mice on day 8 after viral inoculation is shown in Figure 6A. AdipoR1 and AdipoR2 immunoreactivity in myocardial cells from different mice on days 4 and 8 is shown in Figures 6B and 6C. We observed significantly lower AdipoR1 immunoreactivity in myocytes from $\mathrm{OB}$ mice at the same times (Figure 6B). The
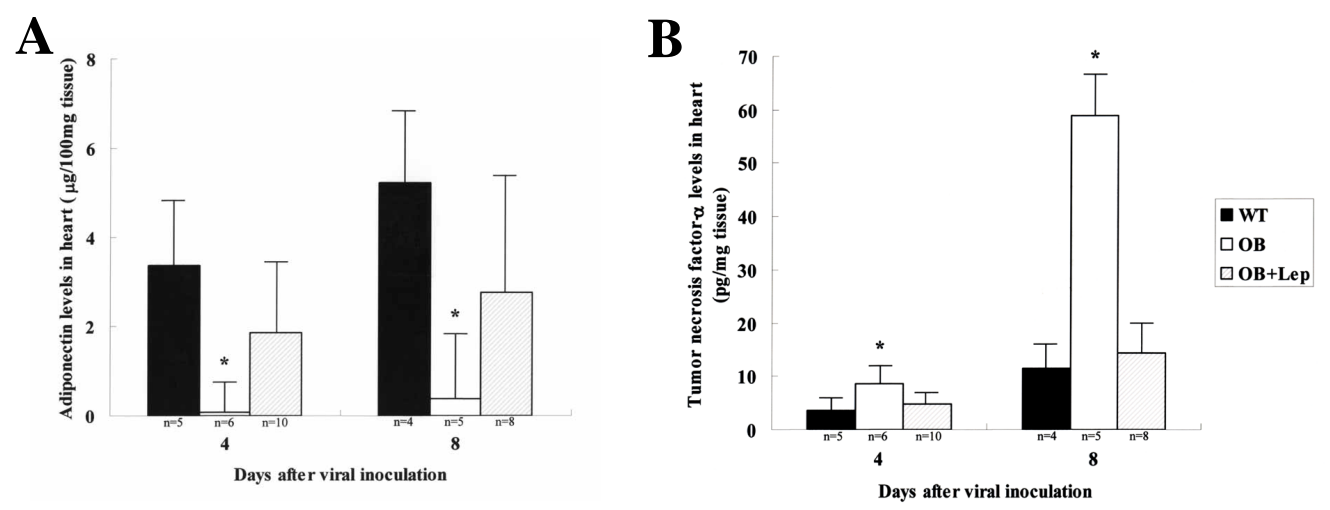

Figure 5. Concentrations of adiponectin (A) and tumor necrosis factor- $\alpha(\mathbf{B})$ in hearts from different mice on days 4 and 8 after viral inoculation. Data are expressed as mean \pm SD. WT indicates wild-type mice; OB, leptin-deficient ob/ob mice; and $\mathrm{OB}+$ Lep, $\mathrm{OB}$ mice receiving daily intraperitoneal administration of mouse leptin (300 $\mu \mathrm{g} / \mathrm{day}$, starting simultaneously with viral inoculation). $* P<0.05$ compared with WT group. 


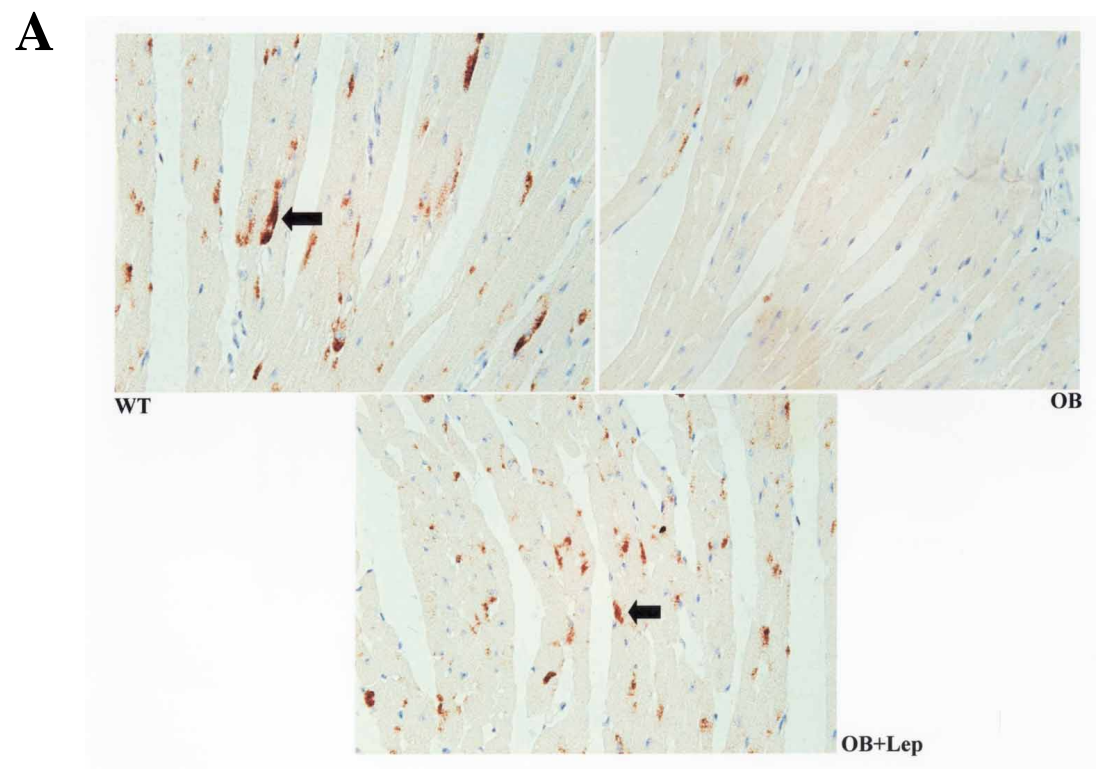

B

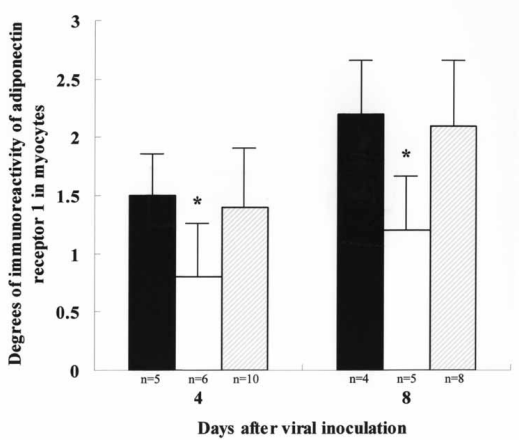

C

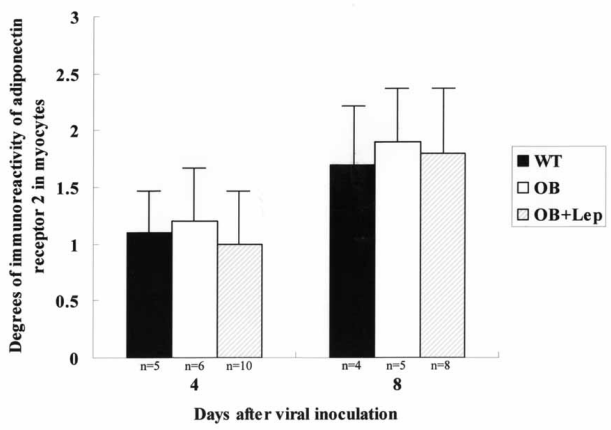

Figure 6. Adiponectin receptor immunoreactivity in cardiomyocytes consisting of photographic demonstration of adiponectin receptor 1 immunoreactivity (arrows) in myocardium from different mice on day 8 after viral inoculation (A, original magnification) and adiponectin receptor 1 (B) or 2 (C) immunoreactivity in myocytes from various mice on days 4 and 8 . Data are expressed as mean \pm SD. WT indicates wild-type mice; OB, leptin-deficient ob/ob mice; and OB + Lep, OB mice receiving daily intraperitoneal administration of mouse leptin $(300 \mu \mathrm{g} / \mathrm{day}$, starting simultaneously with viral inoculation). $* P<0.05$ compared with WT group.

degree of AdipoR1 immunoreactivity in myocytes from OB + Lep mice was similar to that in myocytes from WT mice. There was no significant difference in AdipoR2 immunoreactivity in myocytes among all groups. 


\section{DISCUSSION}

Cardiac expression of adiponectin was impaired in leptin-deficient mice with viral myocarditis. Adiponectin reactivity on immunostaining was recently reported to be observed at the periphery of surviving cardiomyocytes around lesions in the granulation stage in myocardial tissues obtained from 47 autopsied hearts with infarction. ${ }^{20)}$ In another immunohistochemical analysis, the boundary of mouse hepatocytes showed positive signals for adiponectin after 3-6 hours of carbon tetrachloride treatment, and their cytoplasm was intensely stained after 18 hours of treatment. ${ }^{17)}$ Adiponectin was suggested to be produced by the liver in mice, where it underwent tissue damage-induced transcriptional regulation. ${ }^{17)}$ Our data regarding adiponectin expression in surviving myocytes suggest that this adipocytokine may have important implications in the acute phase of viral myocarditis.

Adiponectin is reported to be involved in ending inflammatory responses through its inhibitory actions. ${ }^{21)}$ This cytokine increases mRNA expression of the anti-inflammatory molecule interleukin-10 (IL-10) at the transcriptional level, and promoted IL-10 protein secretion in an in vitro experiment in human monocyte-derived macrophages. ${ }^{22)}$ In addition, a reciprocal relationship between adiponectin and highly-sensitive C-reactive protein was shown in both human plasma and adipose tissue from subjects with coronary artery disease. ${ }^{18)}$ In our experiment, suppressed expression of cardiac adiponectin mRNA, immunoreactivity, and protein in OB mice was associated with the development of severe myocarditis, whereas increased expression of adiponectin in the hearts from WT mice and $\mathrm{OB}+$ Lep mice led to inhibition of the myocardial inflammatory process. Therefore, we speculate that local expression of adiponectin in the damaged heart may be a compensatory phenomenon against the severe inflammatory condition of viral myocarditis.

TNF- $\alpha$ mRNA was shown to be detectable in myocardium obtained from subjects with ischemic heart disease (IHD) and dilated cardiomyopathy (DCM) using Northern blot analysis, while there is no evidence for TNF- $\alpha$ gene expression in the nonfailing human heart. ${ }^{1)}$ Immunohistochemical examination also indicated that there was obvious TNF- $\alpha$ immunostaining of cardiomyocytes from patients with IHD and DCM, whereas TNF- $\alpha$ immunoreactivity was not detectable in the nonfailing heart. ${ }^{1)}$ Natriuretic peptides including atrial and B-type peptides are synthesized and secreted by the heart, and play a critical role in cardiovascular homeostasis. ${ }^{23)}$ Similarly to the previous demonstration, identification of adiponectin expression in the myocardium is also a good example of a contribution made by molecular biology to understanding the mechanisms for heart failure compensation. 
Delporte and colleagues ${ }^{24)}$ have recently suggested a causal relationship exists between leptin and adiponectin in $\mathrm{OB}$ mice. The adiponectin content of visceral fat and adiponectin secretion by adipose tissue were blunted in OB mice. Leptin treatment of OB mice increased adiponectin mRNA, adiponectin content, and secretion from visceral fat by $50-80 \%$. Leptin also directly stimulated adiponectin mRNA and secretion from adipocytes. Leptin replacement therapy was reported to restore adipose tissue adiponectin concentration and secretion, at least in part, via direct stimulation of adiponectin gene expression in OB mice. Therefore, we believe that leptin treatment might induce expression of cardiac adiponectin mRNA and content in OB mice with viral myocarditis through a similar mechanism.

We determined the expression levels of a proinflammatory cytokine, TNF- $\alpha$ mRNA, and protein in the heart from different mice on days 4 and 8 after viral inoculation as previously described. ${ }^{8)}$ Significantly increased levels of cardiac TNF- $\alpha$ mRNA and protein on both days were observed in OB mice compared with those in WT mice, together with severe myocarditis and reciprocal changes in adiponectin mRNA and protein. Administration of leptin to OB mice inhibited the progression of severe myocarditis through augmentation of adiponectin mRNA and protein levels and reduced levels of TNF- $\alpha$ mRNA and protein. Adiponectin-knockout mice revealed a high level of TNF- $\alpha$ mRNA in adipose tissue and a high plasma TNF- $\alpha$ concentration. ${ }^{12)}$ Adiponectin was also reported to inhibit TNF- $\alpha$ production in macrophages. ${ }^{21)}$ Therefore, adiponectin expression induced by leptin replacement seems to be able to protect against myocarditis through suppression of expression of TNF- $\alpha$.

Cloning of cDNA encoding two adiponectin receptors (AdipoR1 and AdipoR2) has recently been demonstrated. ${ }^{25)}$ AdipoR1 is located at chromosome 1p36.13-q41, and AdipoR2 is located at chromosome 12p13.31. AdipoR1 and AdipoR2 are expressed ubiquitously in most organs, especially AdipoR1 in skeletal muscle and AdipoR2 in the liver. ${ }^{25)}$ Pancreatic $\beta$ cells were also shown to express adiponectin receptors in a cell culture system. ${ }^{26)}$ These receptors include 7 transmembrane domains and activate signaling molecules such as PPAR- $\alpha$, AMP-activated protein kinase, and mitogen-activated protein kinase. ${ }^{25)}$ Interestingly, we found AdipoR1 and AdipoR2 immunoreactivity as well as adiponectin immunoreactivity in myocardial cells in a mouse model of viral myocarditis. These results indicate that the surviving myocytes may possess an adiponectinautocrine system, which leads to protection against the progression of myocardial inflammation.

Obesity reduces AdipoR1/R2 expression levels and decreases adiponectin sensitivity, which finally leads to insulin resistance. Improved adiponectin sensitivity should serve as a treatment target for obesity-related diseases. ${ }^{27)}$ Replace- 
ment of leptin in $\mathrm{OB}$ mice inhibited the development of severe myocarditis through augmentation of AdipoR1, but not AdipoR2, immunoreactivity in myocytes. We found that adiponectin replacement therapy could attenuate myocardial damage in $\mathrm{OB}$ mice with viral myocarditis. ${ }^{28)}$ Adiponectin replacement also suppressed the development of severe myocarditis by enhancing myocyte AdipoR1, but not AdipoR2, immunoreactivity. Thus, there seems to be selectivity in the adiponectin receptor subtype in myocytes from OB mice. Similarly to our data, it has been demonstrated in another experiment that elevation of liver AdipoR1 expression and reduction in the AdipoR2 expression were observed in obese mice given a combination of leptin and a melanocortin receptor agonist, although there were no alterations in the AdipoR1 or AdipoR2 expression in the mice receiving the melanocortin receptor agonist alone. ${ }^{29)}$ In addition, changes in AdipoR1/R2 expression in skeletal muscle and adipose tissue have been reported in type 2 diabetic patients during PPAR- $\gamma$ agonist therapy. ${ }^{30)}$ AdipoR 1 expression was up-regulated in adipose tissue but down-regulated in muscle by rosiglitazone. On the other hand, AdipoR2 expression was not changed by rosiglitazone in either of the tissues. The increase in adipose tissue AdipoR1 expression with rosiglitazone was associated with increased postprandial triglyceride clearance and increased fasting fatty acid output. AdipoR1 is suggested to play a role in mediating adiponectin effects in specific tissues in relation to insulin sensitization and antiinflammation. It is necessary to evaluate the effects of adiponectin using AdipoR1-knock out mice in the future.

Conclusion: We found reduced expression of adiponectin mRNA, immunoreactivity, and protein levels and decreased immunoreactivity of AdipoR1 in the heart from $\mathrm{OB}$ mice after viral infection, together with increased heart weight, severe myocardial inflammation, and elevated levels of cardiac TNF- $\alpha$ mRNA and protein. Administration of leptin to OB mice resulted in suppression of the development of severe myocarditis through augmentation of adiponectin mRNA, immunoreactivity, and protein levels, elevated AdipoR 1 immunoreactivity in myocytes, and reduced levels of TNF- $\alpha$ mRNA and protein. Our observations suggest that impaired expression of adiponectin in the heart is associated with the development of viral myocarditis through enhancement of TNF- $\alpha$ expression under a leptin-deficient status.

\section{ACKNOWLEDGMENTS}

This study was supported in part by a Grant for Promoted Research from Kanazawa Medical University (S2004-2 and S2005-5), a Grant for Project Research from the HighTechnology Center of Kanazawa Medical University (H2004-7), and the Science Research Promotion Fund of the Promotion and Mutual Aid Corporation for Private Schools of Japan. 


\section{REFERENCES}

1. Torre-Amione G, Kapadia S, Lee J, et al. Tumor necrosis factor-alpha and tumor necrosis factor receptors in the failing human heart. Circulation 1996; 93: 704-11.

2. Yokoyama T, Vaca L, Rossen RD, Durante W, Hazarika P, Mann DL. Cellular basis for the negative inotropic effects of tumor necrosis factor-alpha in the adult mammalian heart. J Clin Invest 1993; 92: 2303-12.

3. Mantzoros CS. The role of leptin in human obesity and disease: a review of current evidence. Ann Intern Med 1999; 130: 671-80. (Review)

4. Miller KK, Parulekar MS, Schoenfeld E, et al. Decreased leptin levels in normal weight women with hypothalamic amenorrhea: the effects of body composition and nutritional intake. J Clin Endocrinol Metab 1998; 83: 2309-12.

5. Murdoch DR, Rooney E, Dargie HJ, Shapiro D, Morton JJ, McMurray JJ. Inappropriately low plasma leptin concentration in the cachexia associated with chronic heart failure. Heart 1999; 82: 352-6.

6. Wallace AM, McMahon AD, Packard CJ, et al. Plasma leptin and the risk of cardiovascular disease in the West of Scotland Coronary Prevention study (WOSCOPS). Circulation 2001; 104: 3052-6.

7. Makris TK, Stavroulakis GA, Krespi PG, et al. Elevated plasma immunoreactive leptin levels preexist in healthy offspring of patients with essential hypertension. Am Heart J 1999; 138: 922-5.

8. Kanda T, Takahashi T, Kudo S, Takeda T, Tsugawa H, Takekoshi N. Leptin deficiency enhances myocardial necrosis and lethality in a murine model of viral myocarditis. Life Sci 2004; 75: 1435-47.

9. Hu E, Liang P, Spiegelman BM. AdipoQ is a novel adipose-specific gene dysregulated in obesity. J Biol Chem 1996; 271: 10697-703.

10. Yamauchi T, Kamon J, Waki H, et al. Globular adiponectin protected ob/ob mice from diabetes and ApoE-deficient mice from atherosclerosis. J Biol Chem 2003; 278: 2461-8.

11. Berg AH, Combs TP, Du X, Brownlee M, Scherer PE. The adipocyte-secreted protein Acrp30 enhances hepatic insulin action. Nat Med 2001; 7: 947-53.

12. Maeda N, Shimomura I, Kishida K, et al. Diet-induced insulin resistance in mice lacking adiponectin/ACRP30. Nat Med 2002; 8: 731-7.

13. Yamauchi T, Kamon J, Minokoshi Y, et al. Adiponectin stimulates glucose utilization and fatty-acid oxidation by activating AMP-activated protein kinase. Nat Med 2002; 8: 1288-95.

14. Chan KC, Chou HH, Wu DJ, Wu YL, Huang CN. Diabetes mellitus has an additional effect on coronary artery disease. Jpn Heart J 2004; 45: 921-7.

15. Saito K, Tobe T, Minoshima S, et al. Organization of the gene for gelatin-binding protein (GBP28). Gene 1999; 229: 67-73.

16. Kanda T, Koike H, Arai M, et al. Increased severity of viral myocarditis in mice lacking lymphocyte maturation. Int J Cardiol 1999; 68: 13-22.

17. Yoda-Murakami M, Taniguchi M, Takahashi K, et al. Changes in expression of GBP28/adiponectin in carbon tetrachloride-administered mouse liver. Biochem Biophys Res Commun 2001; 285: 372-7.

18. Ouchi N, Kihara S, Funahashi T, et al. Reciprocal association of C-reactive protein with adiponectin in blood stream and adipose tissue. Circulation 2003; 107: 671-4.

19. Kanazawa K, Kawashima S, Mikami S, et al. Endothelial constitutive nitric oxide synthase protein and mRNA increased in rabbit atherosclerotic aorta despite impaired endothelium-dependent vascular relaxation. Am J Pathol 1996; 148: 1949-56.

20. Ishikawa Y, Akasaka Y, Ishii T, et al. Changes in the distribution pattern of gelatin-binding protein of $28 \mathrm{kDa}$ (adiponectin) in myocardial remodelling after ischaemic injury. Histopathology 2003; 42: 43-52.

21. Yokota T, Oritani K, Takahashi I, et al. Adiponectin, a new member of the family of soluble defense collagens, negatively regulates the growth of myelomonocytic progenitors and the functions of macrophages. Blood 2000; 96: 1723-32.

22. Kumada M, Kihara S, Ouchi N, et al. Adiponectin specifically increased tissue inhibitor of metalloproteinase-1 through interleukin-10 expression in human macrophages. Circulation 2004; 109: 2046-9.

23. Grepin C, Dagnino L, Robitaille L, Haberstroh L, Antakly T, Nemer M. A hormone-encoding gene identifies a pathway for cardiac but not skeletal muscle gene transcription. Mol Cell Biol 1994; 14: 3115-29. 
24. Delporte ML, El Mkadem SA, Quisquater M, Brichard SM. Leptin treatment markedly increased plasma adiponectin but barely decreased plasma resistin of ob/ob mice. Am J Physiol Endocrinol Metab 2004; 287: E44653.

25. Yamauchi T, Kamon J, Ito Y, et al. Cloning of adiponectin receptors that mediate antidiabetic metabolic effects. Nature 2003; 423: 762-9.

26. Kharroubi I, Rasschaert J, Eizirik DL, Cnop M. Expression of adiponectin receptors in pancreatic $\beta$ cells. Biochem Biophys Res Commun 2003; 312: 1118-22.

27. Kadowaki T, Yamauchi T. Adiponectin and adiponectin receptors. Endocr Rev 2005; 26: 439-51. (Review)

28. Takahashi T, Saegusa S, Sumino H, et al. Adiponectin replacement therapy attenuates myocardial damage in leptin-deficient mice with viral myocarditis. J Int Med Res 2005; 33: 207-14.

29. Bluher S, Ziotopoulou M, Bullen JW Jr, et al. Responsiveness to peripherally administered melanocortins in lean and obese mice. Diabetes 2004; 53: 82-90.

30. Tan GD, Debard C, Funahashi T, et al. Changes in adiponectin receptor expression in muscle and adipose tissue of type 2 diabetic patients during rosiglitazone therapy. Diabetologia 2005; 48: 1585-9. 